\title{
In silico evaluation of the Eucalyptus transcriptome
}

\author{
Renato Vicentini ${ }^{1}$, Flávio T. Sassaki ${ }^{3}$, Marcos A. Gimenes ${ }^{3}$, Ivan G. Maia ${ }^{3}$ and Marcelo Menossi ${ }^{1,2}$ \\ ${ }^{1}$ Universidade Estadual de Campinas, Centro de Biologia Molecular e Engenharia Genética, \\ Campinas, SP, Brazil. \\ ${ }^{2}$ Universidade Estadual de Campinas, Instituto de Biologia, Departamento de Genética e Evolução, \\ Campinas, SP, Brazil. \\ ${ }^{3}$ Universidade Estadual Paulista 'Júlio de Mesquita Filho', Instituto de Biociências, \\ Departamento de Genética, Botucatu, SP, Brazil.
}

\begin{abstract}
The expressed sequence tags (ESTs) produced in the Forests project provide an invaluable opportunity to assess the Eucalyptus transcriptome. Besides providing information on the different proteins produced by this plant, it is possible to infer gene expression profiles because non-normalized cDNA libraries were used. The EST frequency from any gene is correlated to the transcript levels in the tissues from which the cDNA libraries were constructed. The goal of this work was to identify Eucalyptus genes that showed either differential expression pattern or were ubiquitously expressed in the tissues sampled in the Forests project. Six robust statistical tests and very restrictive rules were applied to gain confidence in the in silico data aiming to avoid false positives. Several genes with interesting expression profiles were identified and some of them were validated by RT-PCR.
\end{abstract}

Key words: transcriptome, Eucalyptus, tissue-specific, statistics, differential expression.

Received: May 28, 2004; Accepted: November 29, 2004.

\section{Introduction}

Expressed sequence tags (ESTs) projects are a powerful strategy to discover genes and also to assess their expression profiles. The abundance of ESTs in a cDNA library reflects the expression levels in the tissues used to extract the RNA (Okubo et al., 1992). The estimation of gene expression levels using the frequency of gene transcripts in non-normalized cDNA libraries has been done in several species, such as rice (Ewing et al., 1999), Medicago truncatula (Journet et al, 2002), sugarcane (Falco et al., 2001) and Schistosoma mansoni (Franco et al., 1997).

As in any genomic approach, gene expression profiling using EST counting produces a large amount of data. The use of robust statistical tests is essential in the identification of differentially expressed genes in EST projects. In the past few years several statistical approaches have been described (Audic and Claverie, 1997; Stekel et al., 2000; Greller and Tobin, 1999; Romualdi et al., 2001).

The extensive representation of the Eucalyptus transcriptome in the Forests database is a rich resource

Send correspondence to Marcelo Menossi. Universidade Estadual de Campinas, Instituto de Biologia, Departamento de Genética e Evolução, Caixa Postal 6010, 13083-875 Campinas, SP, Brazil. E-mail: menossi@unicamp.br. for several studies, including the discovery of genes exhibiting tissue-specific or ubiquitous expression. This is, for example, a first step towards the cloning of promoters that can be used for biotechnological purposes. Most transgenic plants obtained so far use the $35 \mathrm{~S}$ promoter, which is expressed in most cell types. In the majority of cases, the use of promoters with specific expression patterns is desirable either to avoid the waste of energy producing proteins of interest in cells that are not relevant or to avoid metabolic dysfunctions. A good example of this approach is the expression of a transcription factor under the control of a stress-inducible promoter in transgenic tomato plants (Lee et al., 2003) which resulted in normal plants with increased tolerance to drought. In contrast, plants expressing the same gene under the control of the contitutive $35 \mathrm{~S}$ promoter were also drought tolerant, but had a dwarf phenotype and reduced fruit set (Hsieh et al., 2002).

In this work, we used an in silico approach to identify genes from Eucalyptus presenting differential expression in the tissues sampled in the Forests project. A set of six statistical tests was applied and several candidate genes were found. The in silico approach was validated by RT-PCR for selected genes and a searchable database containing all the results was built. 


\section{Materials and Methods}

\section{cDNA libraries and pools}

EST data generated by the Forests consortium (https://forests.esalq.usp.br/) were taken from 18 libraries: BK1, CL1, CL2, FB1, LV2, LV3, RT3, RT6, SL1, SL4, SL5, SL6, SL7, SL8, ST2, ST6, ST7 and WD2 (see Table 1). Libraries representing the same organs or plant parts were grouped in eight pools: BK (only BK1), CL (CL1 and CL2), FB (FB1), LV (LV2 and LV3), RT (RT3, RT6), SL (SL1, SL4, SL5, SL6, SL7 and SL8), ST (ST2, ST6 and ST7) and WD (only WD2).

\section{Identification of gene expression patterns}

To compare the expression level in different libraries, the data were normalized by the number of reads in each library. The IDEG.6 software (Romualdi et al., 2001) was used to calculate the values of six different statistical tests aiming to identify differentially expressed genes in multiple tag sampling experiments. The AC, R and GT statistics (Audic and Claverie, 1997; Stekel et al., 2000; Greller and Tobin, 1999), the Fisher's 2 X2 exact test, the 2 X2 $\chi^{2}$ test and general $\chi^{2}$ test were applied on the Forests data. Three of these statistical tests (Fisher 2X2, 2X2 $\chi^{2}$ and AC) are pair wise tests and the estimated gene expression levels are compared between two libraries or pools. The other three are multicomparison statistical tests and allow the simultaneous comparison of all libraries or pools. The analyses were performed with scripts developed in the Perl programming language. A web-based searchable database is available at https://ipe.cbmeg.unicamp.br.

The identification of library- and pool-specific genes was based upon two rules: A) the cluster must have reads from only one library or pool; B) all statistical tests must have a significance threshold of at least 0.05 . Preferentially expressed genes were considered as those having reads from more than one library or pool. The library or pool with the highest number of reads must have statistically different results from all others, considering a 0.05 threshold. To identify ubiquitously expressed genes we considered only those clusters with at least one read from every library. In this case, all statistical tests must have non-significant results.

\section{Gene expression validation}

The in silico expression pattern of selected ESTs was validated by reverse transcription-PCR (RT-PCR). Total RNA was extracted from leaf, stem, flower, fruit and root tissues of Eucalyptus grandis as described by Korimbocus et al., (2002) with minor modifications. RNA was treated with DNase I at $37^{\circ} \mathrm{C}$ for $15 \mathrm{~min}$ prior to use. Ten $\mu \mathrm{g}$ of total RNA was reverse transcribed using oligo $(\mathrm{dT})_{20}$ and Superscript II (Invitrogen, USA) according to the manufacturer's instructions. An aliquot of two $\mu \mathrm{L}$ from the first-strand cDNA synthesis reaction was used for PCR amplification using gene-specific primers: 5' ACCACGAGC TCAAGGTCAAG 3' and 5' CTTCTCGTTCACACCCAC AA 3' (cluster EGEQFB1001C06.g), 5' TTTGGGGAA CAACTGGAGAG 3' and 5' CCATCAGAAACAGCAAAG CA 3' (EGEQRT3002E03.g), 5' TCTCTCGCCTTGTTG GTCTT 3' and 5' CTGGCCCTGGAACAGAGTTA 3' (EGACFB1015B10.g), 5' GGCATGTTCTGTGCATCA TC 3' and 5' ACCACCAGCACCTTTCCTTC 3' (EGA GLV2214H10.g), 5' CGTCTCAATTCAACGCACAC 3' and 5' CCTCCAGAACGAAGCATACC 3' (EGABST22 22G06.g), 5' AAGGGCTCGATAGGGATCAT 3' and 5' GCGTAGGACCCGATGAAGAT 3' (EGEPRT3362H 10.g), 5' GAACCCTCCCCAGTAAATGC 3' and 5' GC CACACAGAGAGCCAAAGT 3' (EGABSL1082B12.g). Samples were heated to $94{ }^{\circ} \mathrm{C}$ for $3 \mathrm{~min}$ and the amplification was done for 30 and 35 cycles at $94{ }^{\circ} \mathrm{C}$ for $30 \mathrm{~s}, 65^{\circ} \mathrm{C}$ for $45 \mathrm{~s}$ and $72^{\circ} \mathrm{C}$ for $1 \mathrm{~min}$, followed by a final extension at $72{ }^{\circ} \mathrm{C}$ for $10 \mathrm{~min}$. Primers based on an EST encoding a glyceraldehyde-3-phosphate dehydrogenase (GAPDH) were used as an internal control for RNA integrity and equal loading. PCR products were electrophoresed on $1 \%$ agarose gels containing ethidium bromide and visualized under UV radiation.

\section{Results and Discussion}

\section{In silico analyses}

A total of 112,857 ESTs from the Forests project were used to infer gene expression patterns in several tissues from Eucalyptus. Expression profiles were evaluated considering either each library individually or eight pools where libraries from similar tissues/organs were grouped (Table 1).

We were interested in the identification of genes that were specifically or preferentially expressed in a particular library or pool, or in those expressed all over the Eucalyptus tissues sampled in the Forests project. A simple approach to identify such genes is to search for clusters containing reads that are exclusive or mainly present in a particular library. However, this approach does not have a statistical validation and will produce a high number of false positives.

To avoid false positives three statistical methods were used in pair wise comparison and another three to compare all samples at the same time. Only the genes that presented significant threshold $(\mathrm{p}<0.05)$ in all statistical tests were considered.

Five clusters were specific to a single library, while 63 were preferentially expressed in a particular library (Figure 1A). A total of nine clusters was found to be specific to a single pool (Figure 1B), and one cluster, EGE QFB1201B08.g, was selected as specific in both library and pool analyses. The number of preferential clusters found in the pool analyses was much higher: 258. This difference may reflect the fact that several libraries represent 
Table 1 - Description of the FOREST libraries and in silico pools.

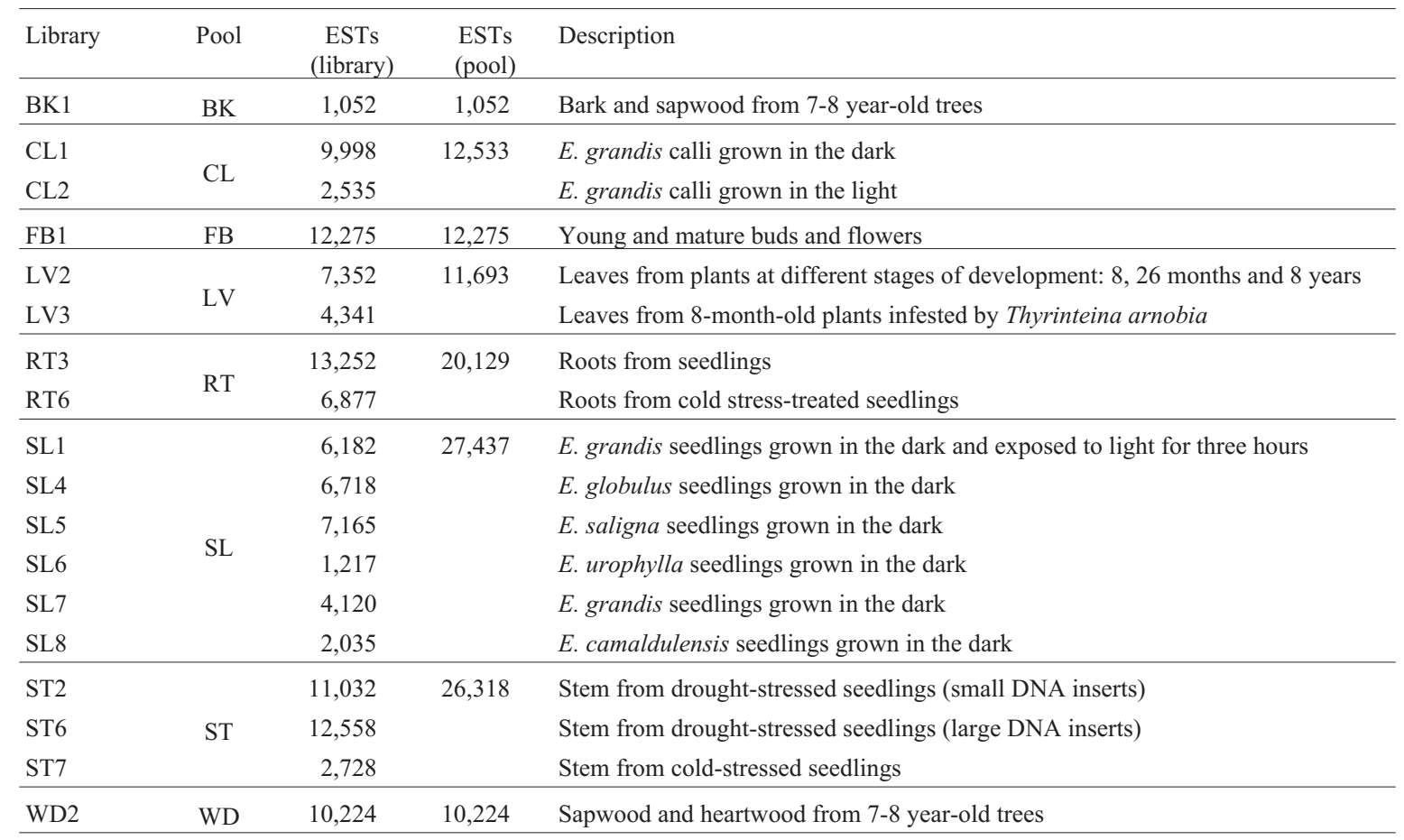

the same tissues/organs taken from different species or grown in slightly different conditions. On the other hand, the small number of genes specific to a library or pool indicates that most genes are expressed in more than one cell type. It is worth noting that the four genes considered specific to the SL1 library were not considered specific to the

A)

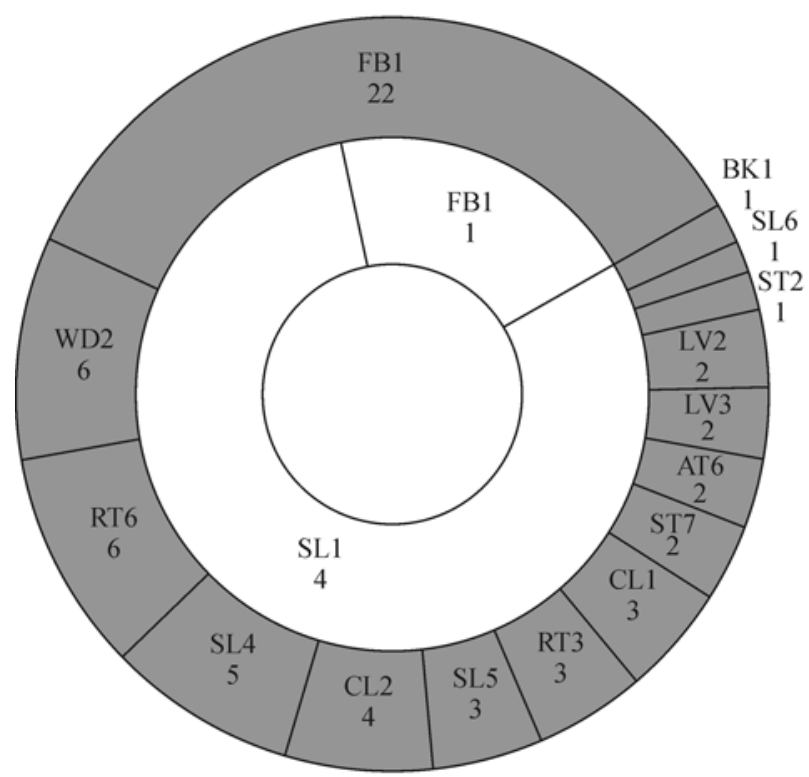

SL pool (Figure 1). This can be understood if we take into account that pools are made from libraries, and consequently the number of elements in each pool is much higher than the number found in each library. So the frequency of ESTs in any particular cluster might drop to a level closer to values expected by chance. As a consequence, the number

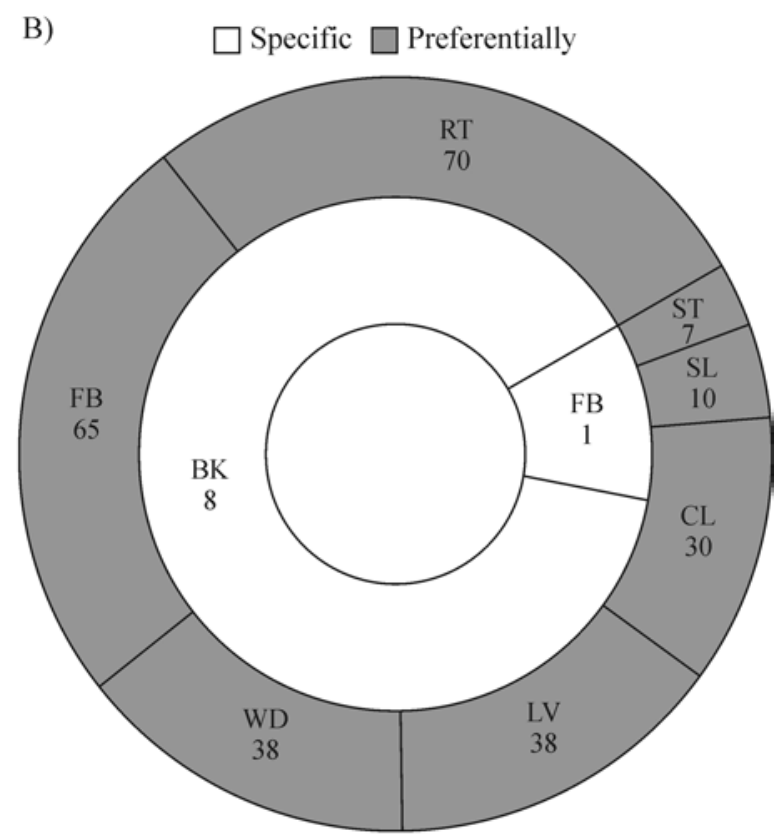

Figure 1 - Distribution of genes with specific or preferential expression in libraries or pools. Six statistical tests were applied to the Forest ESTs and the number of genes with specific or preferential expression in all tests is shown in the inner and outer circles, respectively, for the analysis with libraries (A) or pools of libraries (B). Library and poll names are shown in three and two-character code, respectively, as described in Table 1. 
of genes with significant statistical differences in the analysis of pools is smaller compared with the analysis of libraries.

The number of reads in each library ranged from 1052 (BK1) to 13,252 (RT3) and had no clear correlation with the number of genes with differential expression pattern (Figure 2). This result prompted us to speculate that above a certain threshold of ESTs per library, no significant change in the number of differentially expressed genes is observed. A striking difference was observed for FB1 library, corresponding to flowers, flower buds and fruits, which had the highest number of differentially expressed genes. The pres-

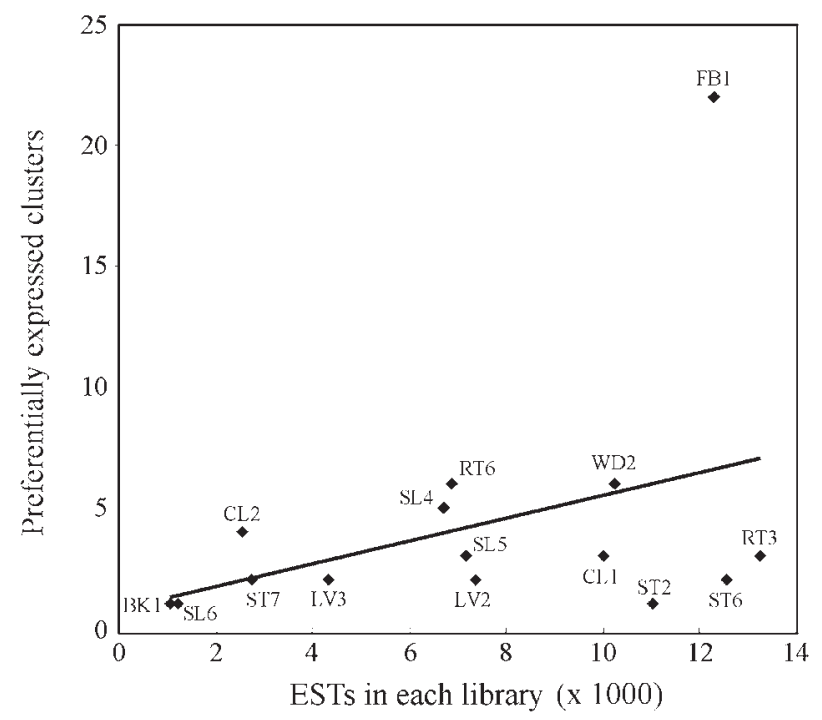

Figure 2 - Distribution of Eucalyptus genes with preferential expression. Each point represents the number of genes with preferential expression in each cDNA library. Libraries are indicated by a three-character code, as described in Table 1. The line represents the regression of the data. ence of a high number of genes preferentially expressed in flower tissues was also observed in silico in sugarcane (Figueiredo et al., 2001), indicating that this organ has special features that require a wide range of protein functions.

The library- and/or pool-specific genes are shown in Table 2. No predominance of any particular protein class could be observed, except for two cytochrome P450. It is interesting to note that five genes encode proteins with unknown function. The normalized expression levels of most clusters (considering the number of ESTs at 10,000) were around 19 and a few clusters had 6-8 ESTs per 10,000 ESTs. The highest value was observed for cluster EGEQ FB1201B08.g, with a normalized expression of 43. This gene encodes a protein with high similarity to two cytochrome P450-dependent enzymes that were induced by wounding in peas (Frank et al., 1996) and by elicitors in soybeans (Schopfer and Ebel, 1998), making the gene an attractive target for further studies. Moreover, the expression value based in EST counting can be used to infer the strength of the corresponding promoters. However, it is worth noting that some genes may be subject to posttranscriptional regulation, altering mRNA stability, for example, which would mask the results. In the case of genes with low expression levels, a strategy to increase the transcriptional activity without losing the expression pattern is to use multiple copies of the promoter, as observed in the stress inducible HAV22 promoter from barley (Lee et al., 2003).

Due to the high number of genes that had preferential expression in libraries or pools, only the most expressed ones (per library or pool) are shown (Table 3). The range of normalized expression varied from 5 to 31 (mean of 11) in libraries and from 1.8 to 31 (mean 4.24) in pools. No predominance of any type of protein could be observed. Sur-

Table 2 - Library- and pool-specific clusters. The number of ESTs in each cluster and the normalized expression (corrected for 10,000 ESTs per library) are indicated. The best hit protein was identified by Blast using a threshold E-values of $\leq \mathrm{e}^{-5}$ (Altshul et al., 1997). The p-value indicates the highest threshold observed in the different statistical tests.

\begin{tabular}{|c|c|c|c|c|c|}
\hline Cluster & Library or pool & ESTs & Normalized expression & Best hit protein & $\mathrm{p}$-value \\
\hline EGEQBK1114A01.g & BK & 3 & 28.52 & unknown & 0.001 \\
\hline EGEQBK1002H06.g & & 2 & 19.01 & cytochrome P450 & 0.01 \\
\hline EGEQBK1086G10.g & & 2 & 19.01 & no hit & 0.01 \\
\hline EGEQBK1088F04.g & & 2 & 19.01 & tonB-dependent receptor & 0.01 \\
\hline EGEQBK1500C09.g & & 2 & 19.01 & transglutaminase-like enzymes & 0.01 \\
\hline EGJMBK1144B02.g & & 2 & 19.01 & glutaredoxin family protein & 0.01 \\
\hline EGUTBK1007E12.g & & 2 & 19.01 & putative p23 co-chaperone & 0.01 \\
\hline EGUTBK1011F06.g & & 2 & 19.01 & putative sugar transporter & 0.01 \\
\hline EGEQFB1201B08.g & FB1 & 53 & 43.18 & cytochrome P450 & 0.00001 \\
\hline EGBMSL1091A09.g & SL1 & 5 & 8.09 & putative protein kinase & 0.01 \\
\hline EGEQSL1055F04.g & & 5 & 8.09 & no hit & 0.01 \\
\hline EGABSL1068F03.g & & 4 & 6.47 & putative protein & 0.05 \\
\hline EGABSL1081E10.g & & 4 & 6.47 & hypothetical protein & 0.05 \\
\hline
\end{tabular}


Table 3 - Clusters preferentially expressed in libraries or pools. The number of ESTs in each cluster and the normalized expression (corrected for 10,000 ESTs per library) are indicated. The best hit protein was identified by Blast using a threshold E-values of $\leq \mathrm{e}^{-5}$ (Altshul et al., 1997). The p-value indicates the highest threshold observed in the different statistical tests.

\begin{tabular}{|c|c|c|c|c|c|}
\hline Cluster & $\begin{array}{l}\text { Library or } \\
\text { pool }\end{array}$ & ESTs & $\begin{array}{l}\text { Normalized } \\
\text { expression }\end{array}$ & Best hit protein & p-value \\
\hline EGBMCL1290G11.g & $\mathrm{CL}$ & 14 & 11.17 & transferase family protein & 0.0001 \\
\hline EGBMCL1290E03.g & CL1 & 7 & 7.00 & seven transmembrane MLO family protein & 0.0001 \\
\hline EGJMCL2028D04.g & CL2 & 4 & 15.78 & lysine and histidine specific transporter & 0.0001 \\
\hline EGCEFB1021A04.g & FB1 and FB & 38 & 30.96 & No hit & 0.00001 \\
\hline EGEQLV2201B04.g & LV & 10 & 8.55 & auxin-binding protein & 0.00001 \\
\hline EGCCLV2224H06.g & LV2 & 7 & 9.52 & early light-inducible protein & 0.0001 \\
\hline EGSBLV3292G03.g & LV3 & 6 & 13.82 & catechol O-methyltransferase & 0.001 \\
\hline EGEQRT3301H05.g & RT3 and RT & 12 & 9.06 & oxidoreductase & 0.00001 \\
\hline EGCCRT6008F08.g & RT6 & 6 & 8.72 & ubiquitin & 0.01 \\
\hline EGEPSL4227F09.g & SL4 and SL & 9 & 13.40 & alkaline alpha galactosidaseII & 0.0001 \\
\hline EGCBSL5004B06.g & SL5 & 5 & 6.98 & acetyltransferase & 0.05 \\
\hline EGUTSL6223C07.g & SL6 & 3 & 24.65 & putative cinnamyl-alcohol dehydrogenase & 0.00001 \\
\hline EGCEST2257B10.g & ST2 & 6 & 5.44 & putative arm repeat protein & 0.01 \\
\hline EGJMST6256E09.g & ST6 and ST & 7 & 5.57 & putative trypanothione-dependent peroxidase & 0.01 \\
\hline EGRFST7254A06.g & ST7 & 4 & 14.66 & sulfate transporter & 0.00001 \\
\hline EGEQWD2247D05.g & WD2 and WD & 17 & 16.63 & dioxygenase-related & 0.00001 \\
\hline
\end{tabular}

prisingly, one cluster encoding a putative ubiquitin (EGC CRT6008F08.g) preferentially expressed in roots was found.

Genes that are expressed in most tissues are good targets to clone ubiquitous promoters that can be useful in some applications. The selection of ubiquitous candidates was based in two assumptions: the cluster must have reads in every library and no significant statistical difference should be detected among any comparison. Eight clusters were in agreement with these conditions (Table 4). The in silico expression pattern of these genes is shown in Figure 3. Two of them, EGEQRT3100H05.b and EGEQRT $3201 \mathrm{C} 10 . \mathrm{g}$, encode proteins with similarity to the alpha subunit of the translation elongation factor 1 , which is encoded by well-known ubiquitously expressed genes.

Table 4 - Ubiquitously expressed clusters. The best hit protein was identified by Blast using a threshold E-values of $\leq \mathrm{e}^{-5}$ (Altshul et al., 1997).

\begin{tabular}{ll}
\hline Cluster & Best hit protein \\
\hline EGEQFB1001H11.g & catalase \\
EGEQFB1002E09.g & aquaporin \\
EGEQLV2002E04.g & aluminum induced protein \\
EGEQRT3002C11.g & chaperone Hsp90-2 \\
EGEQRT3100H05.b & EF-1 alpha \\
EGEQRT3200G03.g & DnaJ protein \\
EGEQRT3201C10.g & EF-1 alpha \\
EGEQRT5002G11.g & heat shock protein \\
\hline
\end{tabular}

In these in silico analyses, multiple statistical tests and very restrictive rules were applied to avoid false positives. We are aware that many false negatives will be present, leaving open the possibility that several other genes represented in the Forests database probably have interesting expression patterns when less restrictive parameters are applied. That was the case when libraries CL (callus) and SL (seedlings) were not considered in the analyses and the same rules as described above were applied. Since both libraries are a mixture of tissues, they are redundant to other cDNA libraries. The consequence is that several genes specific or preferentially expressed in a library made from a particular tissue or organ would not be considered in the analyses if they were also found in CL or SL libraries.

The effects of ignoring these two libraries were clear: the number of genes specifically or preferentially expressed in one library increased from 5 to 31 and from 63 to 9,735 , respectively (Figure 4). The genes specific to a particular pool were unchanged, whereas the total number of genes preferentially expressed in a particular pool increased to 4,745 . The description of these genes can be found in the supplementary material web page at http://ipe.cbmeg. unicamp.br/pub/sup/. These findings indicate that for any particular goal, it is important to carefully set the parameters to assess the genes with useful expression patterns. Considering that all the data from this work is stored in a searchable, web-based database, any particular task can be easily done. 

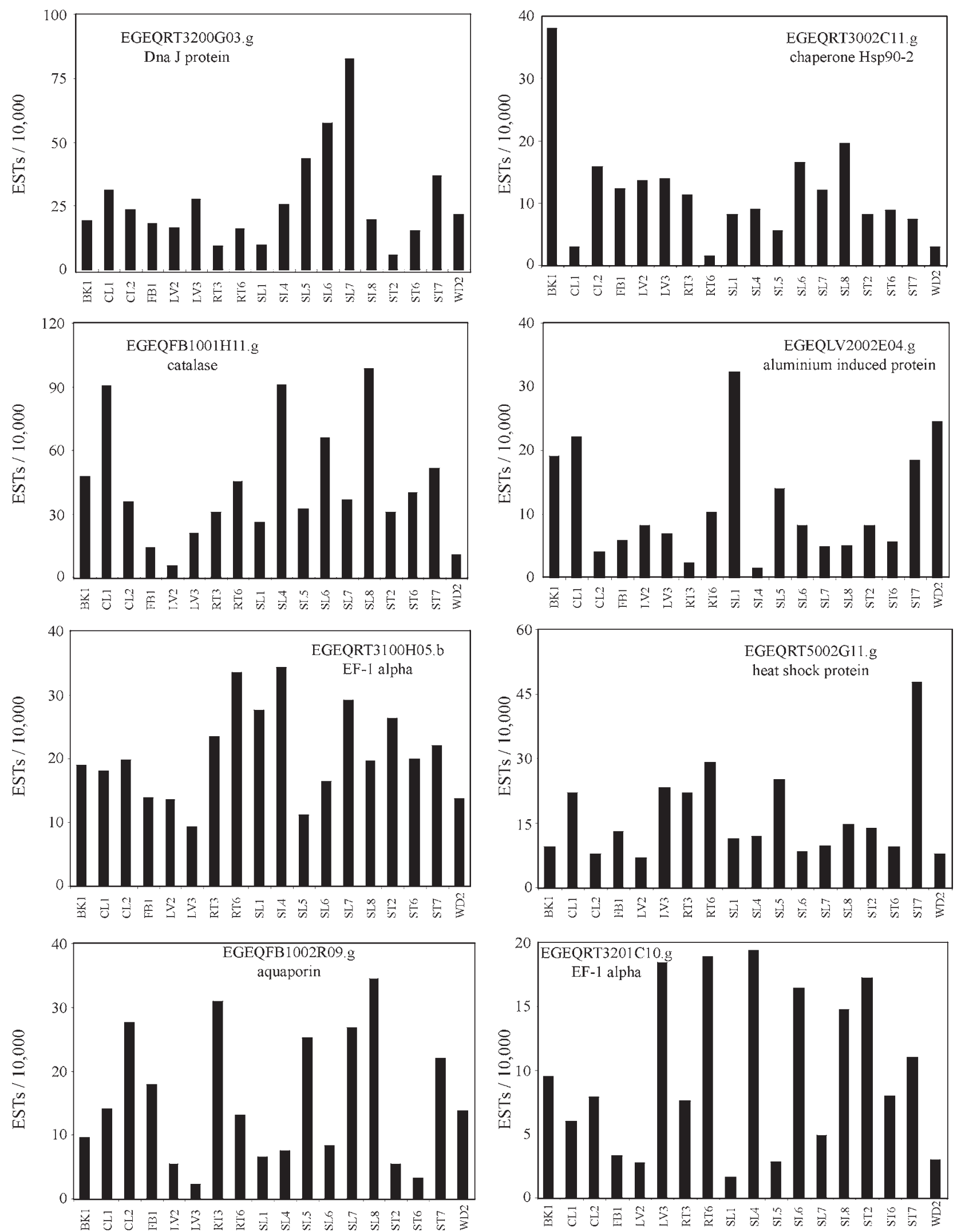

Figure 3 - In silico expression profile of ubiquitous clusters. The EST frequency was normalized by the number of reads in each library and corrected to 10,000 ESTs. Libraries are indicated by a three-character code, as described in Table 1 . The cluster name and the annotation based in the Blast hits are shown for each gene. 
A)

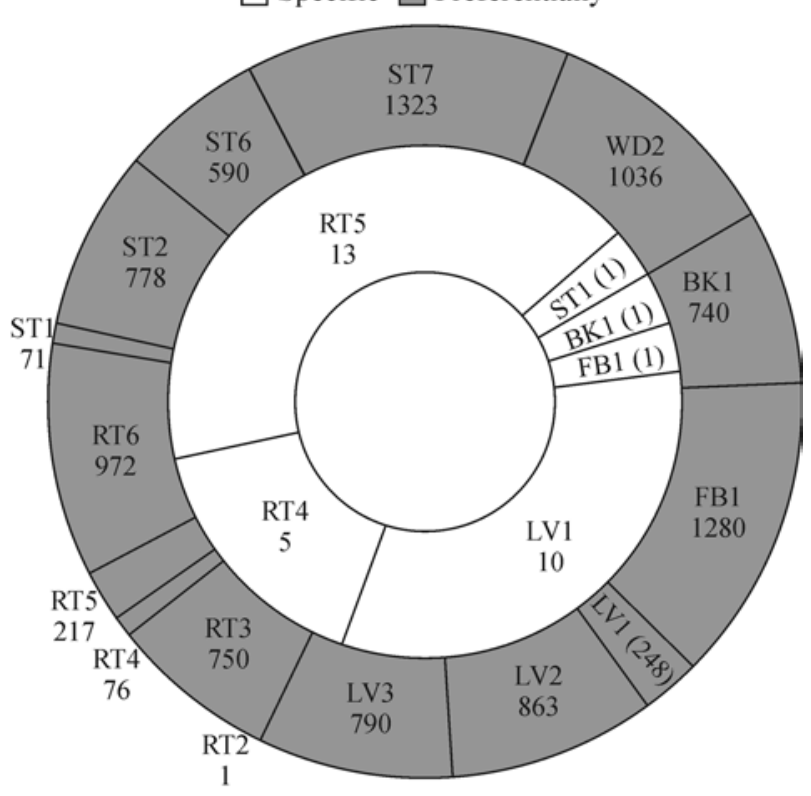

B)

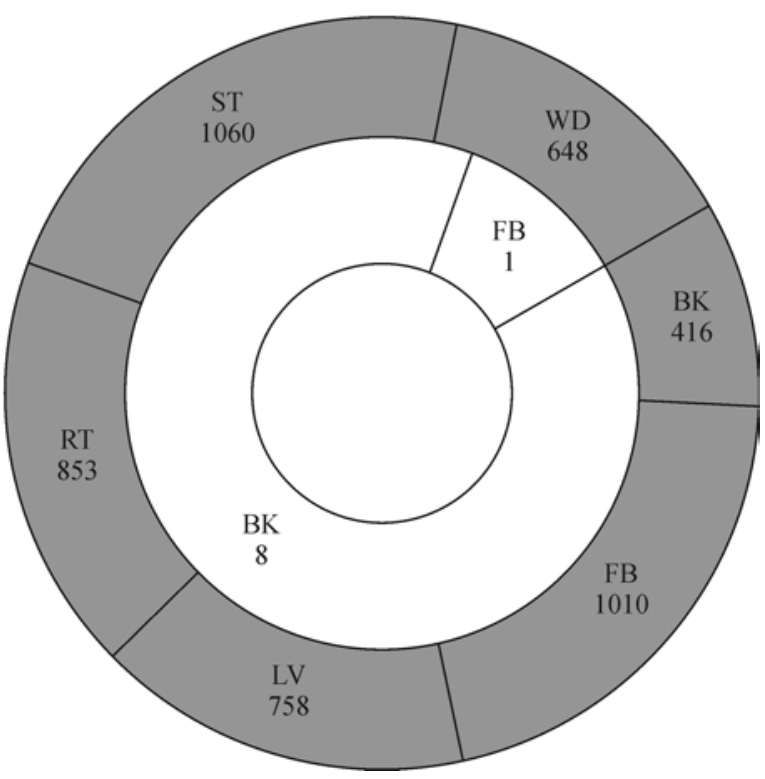

Figure 4 - Distribution of genes with specific or preferential expression in libraries or pools when calli and seedlings libraries and pools were not considered. Six statistical tests were applied to the Forest ESTs and the number of genes with specific or preferential expression in all tests is shown in the inner and outer circles, respectively, for the analysis with libraries (A) or pools of libraries (B). Library and poll names are shown in three- and two-character code, respectively, as described in Table 1.

\section{Validation of the in silico expression pattern}

The expression profile of selected genes was evaluated by semi-quantitative RT-PCR using total RNA extracted from leaf (organ used in LV libraries), stem (ST libraries), flower and fruit (both in the FB1 library, that also had flower buds) and root tissues (RT libraries) of Eucalyptus grandis.

According to the in silico tests, the cluster EGACFB 1015B10.g had preferential expression in the FB library and the FB pool. The expression profile found by RT-PCR confirmed the in silico data, and the corresponding transcripts were detected only in flower buds (Figure 5A). Curiously, this gene was not expressed in fruits, an organ that was also sampled in the FB library. This gene encodes a putative protein that probably represents a novel Eucalyptus gene since it presented no similarity to database entries.

Cluster EGEPRT3362H10.g encodes a polypeptide with high similarity to TIPs (tonoplast intrinsic proteins) and had reads both in RT and SL pools. Although in these two pools an identical estimated expression level of the $\mathrm{Eu}$ calyptus TIP gene was observed, a homologous gene from maize presents a root-specific expression (Lopez et al., 2004). Based on this finding, we decided to check whether the TIP gene corresponded to a false negative in the in silico analyses. The RT-PCR evaluation showed that TIP transcripts were detected only in roots, confirming our suspicion (Figure 5A). As stated above, since the in silico approach was designed to prevent false positives, a large number of false negatives should be expected. This highlights the hypothesis that several genes not considered as specific or preferential in this study may have interesting expression patterns.

The deduced protein from cluster EGEQRT3002 E03.g had significant similarity to BOR1, an efflux-type boron transporter for xylem loading in Arabidopsis thaliana (Takano et al., 2002). The Eucalyptus BOR1 gene presented four reads from the RT pool and one read from the CL pool (data not shown). By simple EST counting, this cluster might be considered as preferentially expressed in roots although our statistical tests did not support this inference. In agreement with the statistical test, the RT-PCR assay supported the hypothesis that this gene was not preferentially expressed in roots. Although expressed in roots, similar levels of transcripts were also observed in other organs (Figure 5A) as reported for the A. thaliana $B O R 1$ gene, which is expressed in roots and shoots (Takano et al., 2002). The possible expression of the Eucalyptus $B O R-1$ in undifferentiated tissues could therefore explain the observed expression pattern.

Cluster EGABSL1082B12.g also displayed no specific or preferential expression in the RT-PCR assay (Figure $5 \mathrm{~A}$ ). This cluster, encoding a putative protein, was enriched in reads from pool SL but was not granted by the statistical tests. This was also the case for the other two clusters, EGAGLV2214H10.g, enriched in reads from pool LV and encoding an homologue of the pea Cytochrome B6-F complex iron-sulfur subunit (Salter et al., 1992), and EGABST2222G06.g, enriched in reads from pool BK and encoding a protein with high identity to beta-carotene hydroxylases from Citrus unshiu (Kim et al., 2001) (data 


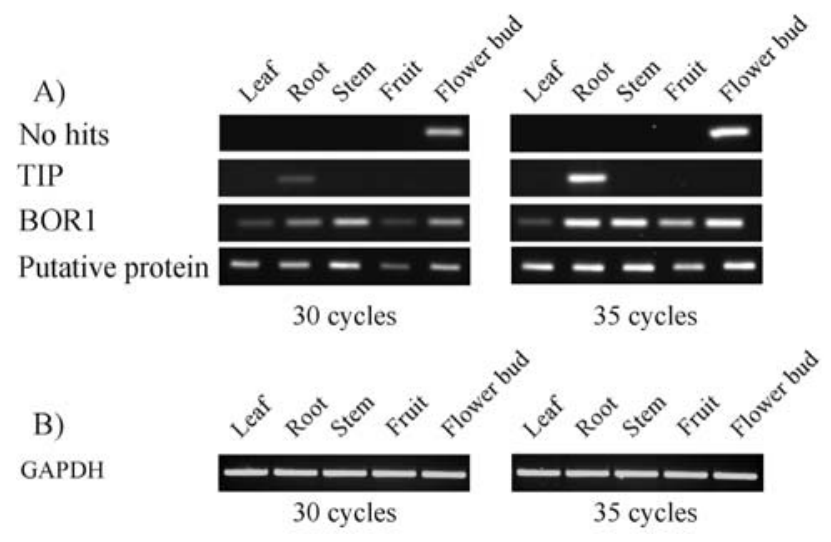

Figure 5 - RT-PCR validation of candidate clusters. RT-PCR was performed with specific primers using RNA from the organs indicated above each lane and the products were separated in an $1 \%$ agarose gel containing ethidium bromide. A) Candidate genes with possible organ-specific or -preferential expression, encoding the following proteins. The deduced protein was named according to the best Blast hit: No hits, without homology in the database (cluster EGACFB1015B10.g); TIP (EGEPRT3362H10.g), BOR1 (EGEQRT3002E03.g) and a Putative protein (EGABSL1082B12.g). (B) GAPDH control (EGEQFB1001C06.g). Numbers at the bottom of each panel indicate the number of reaction cycles.

not shown). These results reinforce our view that the use of multiple statistical tests is a good strategy to prevent false positives.

The EGEQFB1001C06.g cluster encodes a glyceraldehyde-3-phosphate dehydrogenase (GAPDH). Genes encoding these proteins are widely used as control in gene expression studies because they are ubiquitously and constitutively expressed. However, the Eucalyptus GAPDH gene did not pass in the in silico tests because it has no reads in the BK pool. When this pool was not included in the analyses, this cluster was considered ubiquitous. The expression pattern deduced from the RT-PCR assay confirmed the expression in all organs at similar levels (Figure 5B), indicating that the Eucalyptus GAPDH gene is indeed ubiquitously expressed.

\section{Outlook for the Future}

The in silico analysis presented here constitutes a valuable tool for predicting expression patterns for a large number of ESTs. We hope this bioinformatics tool will be of great help to many studies, especially those targeting promoter identification and cloning.

\section{Acknowledgements}

IGM and MM received a Research Fellowship from Conselho Nacional de Desenvolvimento Científico e Tecnológico (CNPq). This work was supported by Fundação de Amparo à Pesquisa do Estado de São Paulo (FAPESP), with grant $03 / 00724-7$ to I.G.M.

\section{References}

Altschul SF, Madden TL, Schäffer AA, Zhang J, Zhang Z, Miller W and Lipman DJ (1997) Gapped BLAST and PSI-BLAST: A new generation of protein database search programs. Nucleic Acids Res 25:3389-3402.

Audic S and Claverie JM (1997) The significance of digital gene expression profiles. Genome Res 7:986-995.

Ewing RM, Ben Kahla A, Poirot O, Lopez F, Audic S and Claverie JM (1999) Large-scale statistical analyses of rice ESTs reveal correlated patterns of gene expression. Genome Res 9:950-959.

Falco MC, Marbach PAS, Pompermayer P, Lopes FCC and Silva-Filho MC (2001) Mechanisms of sugarcane response to herbivory. Genet Mol Biol 24:113-122.

Figueiredo RC, Brito MS, Figueiredo LHM, Quiapin AC, Vitorelli PM, Silva LR, Santos RV, Molfetta JB, Goldman GH and Goldman MHS (2001) Dissecting the sugarcane expressed sequence tag (SUCEST) database: Unraveling flower-specific genes. Genet Mol Biol 24:77-84.

Franco GR, Rabelo EM, Azevedo V, Pena HB, Ortega JM, Santos TM, Meira WS, Rodrigues NA, Dias CM, Harrop R, Wilson A, Saber M, Abdel-Hamid H, Faria MS, Margutti ME, Parra JC and Pena SD (1997) Evaluation of cDNA libraries from different developmental stages of Schistosoma mansoni for production of expressed sequence tags (ESTs). DNA Res 4:231-40.

Frank MR, Deyneka JM and Schuler MA (1996) Cloning of phenylpropanoid pathway P450 monooxygenases expressed in Pisum sativum. Plant Physiol 110:1035-1046

Greller LD and Tobin FL (1999) Detecting selective expression of genes and proteins. Genome Res 9:282-296.

Hsieh TH, Lee JT, Charng YY and Chan MT (2002) Tomato plants ectopically expressing Arabidopsis CBF1 show enhanced resistance to water deficit stress. Plant Physiology 130:618-626.

Journet EP, van Tuinen D, Gouzy J, Crespeau H, Carreau V, Farmer M-J, Niebel A, Schiex T, Jaillon O, Chatagnier O, Godiard L, Micheli F, Kahn D, Gianinazzi-Pearson V and Gamas P (2002) Exploring root symbiotic programs in the model legume Medicago truncatula using EST analysis. Nucleic Acids Res 30:5579-5592.

Kim IJ, Ko KC, Kim CS and Chung WI (2001) Isolation and characterization of cDNAs encoding beta-carotene hydroxylase in Citrus. Plant Sci 161:1005-1010.

Korimbocus J, Coates D, Barker I and Boonham N (2002) Improved detection of Sugarcane yellow leaf virus using a real-time fluorescent (TaqMan) RT-PCR assay. J Vir Methods 103:109-120.

Lee JT, Prasad V, Yang PT, Wu JF, David Ho, TH, Charng YY and Chan MT (2003) Expression of Arabidopsis CBF1 regulated by an $\mathrm{ABA} /$ stress inducible promoter in transgenic tomato confers stress tolerance without affecting yield. Plant Cell Environ 26:1181-1190.

Lopez F, Bousser A, Sissoeff I, Hoarau J and Mahe A (2004) Characterization in maize of ZmTIP2-3, a root-specific tonoplast intrinsic protein exhibiting aquaporin activity. J Exp Bot 55:539-541.

Okubo K, Hori N, Matoba R, Niiyama T, Fukushima A, Kojima Y and Matsubara K (1992) Large scale cDNA sequencing for analysis of quantitative and qualitative aspects of gene expression. Nat Genet 2:173-179. 
Romualdi C, Bortoluzzi S and Danieli GA (2001) Detecting differentially expressed genes in multiple tag sampling experiments: Comparative evaluation of statistical tests. Human Mol Genet 10:2133-2141.

Salter AH, Newman BJ, Napier JA and Gray JC (1992) Import of the precursor of the chloroplast Rieske iron-sulphur protein by pea chloroplasts. Plant Mol Biol 20:569-574.

Schopfer CR and Ebel J (1998) Identification of elicitor-induced cytochrome P450s of soybean (Glycine max L.) using differential display of mRNA. Mol Gen Genet 258:315-322.
Stekel DJ, Git Y and Falciani F (2000) The comparison of gene expression from multiple cDNA libraries. Genome Res 10:2055-2061.

Takano J, Noguchi K, Yasumori M, Kobayashi M, Gajdos Z, Miwa K, Hayashi H, Yoneyama T and Fujiwara T (2002) Arabidopsis boron transporter for xylem loading. Nature 420:337-340.

Associate Editor: Marcio de Castro Silva Filho 